\title{
STATISTICAL RESOLUTION LIMIT FOR MULTIPLE PARAMETERS OF INTEREST AND FOR MULTIPLE SIGNALS
}

\author{
Mohammed Nabil El Korso, Remy Boyer, Alexandre Renaux and Sylvie Marcos \\ Laboratoire des Signaux et Systémes (L2S) \\ Université Paris-Sud XI (UPS), CNRS, SUPELEC, \\ Gif-Sur-Yvette, France \\ \{elkorso, remy.boyer, alexandre.renaux, marcos\}@1ss.supelec.fr
}

\begin{abstract}
The concept of Statistical Resolution Limit (SRL), which is defined as the minimal separation to resolve two closely spaced signals, is an important tool to quantify performance in parametric estimation problems. This paper generalizes the SRL based on the Cramér-Rao bound to multiple parameters of interest per signal and for multiple signals. We first provide a fresh look at the SRL in the sense of Smith's criterion by using a proper change of variable formula. Second, based on the Minkowski distances, we extend this criterion to the important case of multiple parameters of interest per signal and to multiple signals. The results presented herein can be applied to any estimation problem and are not limited to source localization problems.
\end{abstract}

Index Terms - Statistical resolution limit, performance analysis, Cramér-Rao bound.

\section{INTRODUCTION}

Characterizing the ability of resolving closely spaced signals is an important step to quantify estimators performance. The concept of Statistical Resolution Limit (SRL), i.e., the minimum distance between two closely spaced signals that allows a correct resolvability, is rising in several applications (especially in parameter estimation problems such as radar, sonar, spectral estimation [1] etc.) There essentially exist two approaches to obtain a SRL: (1) the first is based on the estimation accuracy $[2,3]$ while $(2)$ the second is based on the detection theory [4]. In this paper we consider the SRL based on the estimation accuracy. The Cramér-Rao Bound (CRB) does not directly point out the best resolution that can be achieved by an unbiased estimator. However, since it expresses a lower bound on the covariance matrix of any unbiased estimator, it can be used to obtain the SRL. We distinguish two main criteria on the SRL based on the CRB. The first one was introduced by Lee in [2]: two signals (for example parameterized by the Direction Of Arrivals (DOA) $\theta_{1}$ and $\theta_{2}$ ) are said to be resolvable w.r.t. the DOA if the maximum standard deviation is less than twice the difference between $\theta_{1}$ and $\theta_{2}$. Assuming that the CRB is a tight bound (under mild conditions), the standard deviation, $\sigma_{\theta_{1}}$ and $\sigma_{\theta_{2}}$, of an unbiased estimator can be approximated by $\sqrt{\mathrm{CRB}\left(\theta_{1}\right)}$ and $\sqrt{\mathrm{CRB}\left(\theta_{2}\right)}$, respectively. Consequently, the SRL $\delta_{\theta}$ is defined, in Lee's criterion sense, as $2 \max \left\{\sqrt{\operatorname{CRB}\left(\theta_{1}\right)}, \sqrt{\operatorname{CRB}\left(\theta_{2}\right)}\right\}$. Lee [2] and Dilaveroglu [5] used this criterion to obtain the SRL of frequency estimates. Swingler [6]

This project is funded by both the Région Île-de-France and the Digiteo Research Park. used the same criterion for close frequencies in the case of complex spaced sinusoids. However, the main problem of this criterion is that the coupling between parameters is ignored. To overcome this problem, Smith [3] proposed the following criterion: two signals are resolvable w.r.t. the DOA if the difference between the DOA is greater than the standard deviation of the DOA difference estimation according to the $C R B$. Consequently, the SRL, in Smith's criterion sense, is defined as $\delta_{\theta}$ for which $\delta_{\theta}<\sqrt{\mathrm{CRB}\left(\delta_{\theta}\right)}$ is achieved. This means that, the SRL is obtained by resolving the implicit equation $\delta_{\theta}^{2}=\mathrm{CRB}\left(\delta_{\theta}\right)$. In [7], an example of study of the SRL for DOA of discrete signals based on Smith's criterion has been considered.

In several estimation problems, the signals are parameterized by more than one parameter of interest per signal, for example in the context of, near-field source localization [8] (bearing, elevation and range), polarized source [9] (DOA and the polarization state parameters) and more generally in communication applications [10]. However, Lee and Smith's criteria were introduced only when the signal is parameterized by only one parameter (for example frequency, DOA etc.) To the best of our knowledge, no results are avalaible on the extension of the SRL to multiple parameters of interest per signal. Thus, the aim of this paper is to fill this lack. We first begin by giving a fresh look at Smith's criterion using a proper change of variable formula. Then we show that the extension to the multiple parameters per signal case is not straighforward. Finally, we propose an extension to the case of multiple parameters of interest and multiple signals using the $k$-norm distance. One should note that, the SRL presented herein can be applied to any estimation problem and is not limited to the source localization problem.

\section{PROBLEM SETUP AND BACKGROUND}

The observation model for $M$ signals following the waveform described by the functional $\boldsymbol{f}($.$) is given by$

$$
\mathbf{x}=\sum_{m=1}^{M} \boldsymbol{f}\left(\boldsymbol{\xi}_{m}\right)+\mathbf{n},
$$

where $\mathbf{n}$ denotes the additive noise. The parameters are collected in $\overline{\boldsymbol{\xi}}=\left[\boldsymbol{\xi}_{1}^{T} \ldots \boldsymbol{\xi}_{M}^{T}\right]^{T}$, with a proper rearrangement of $\overline{\boldsymbol{\xi}}$ one can obtain $\boldsymbol{\xi}=\left[\begin{array}{ll}\boldsymbol{\omega}^{T} & \boldsymbol{\rho}^{T}\end{array}\right]^{T}$ where $\boldsymbol{\omega}$ is the $(M P) \times 1$ vector of the parameters of interest and $\rho$ denotes the vector obtained by concatenation of the unwanted and nuisance parameters. This means that we consider $P$ parameters of interest for each signal. To the best of our knowledge, the state of art [3] tackles this problem only in the case of $M=2$ and $P=1$. The problem addressed herein is to derive the Statistical Resolution Limit (SRL) based on the Cramér-Rao Bound (CRB) in 
the case of $P \geq 1$ and $M \geq 2$.

First, let us consider the SRL for two impinging signals w.r.t. one parameter of interest per signal. Consequently, the vector of the parameters of interest is given by $\boldsymbol{\omega}=\left[\begin{array}{ll}\omega_{1} & \omega_{2}\end{array}\right]^{T}$, where we assume that $\omega_{1} \neq \omega_{2}$. Under mild conditions, $E\left\{\left([\hat{\boldsymbol{\xi}}]_{i}-[\boldsymbol{\xi}]_{i}\right)^{2}\right\} \geq$ $[\mathbf{C R B}(\boldsymbol{\xi})]_{i, i}$ where $\hat{\boldsymbol{\xi}}$ denotes an unbiased estimator of $\boldsymbol{\xi}$ and $\operatorname{CRB}(\boldsymbol{\xi})=\mathbf{F I M}^{-1}(\boldsymbol{\xi})$, in which $\operatorname{FIM}(\boldsymbol{\xi})$ denotes the Fisher Information Matrix for model (1) regarding to $\xi$ [11]. In the following, for sake of simplicity, the notation $\mathbf{C R B}\left([\boldsymbol{\xi}]_{1: i}\right)$ will be used instead of the Matlab notation $[\mathbf{C R B}(\boldsymbol{\xi})]_{1: i, 1: i}$.

Having $\mathbf{C R B}(\boldsymbol{\xi})$, one can deduce $\mathbf{C R B}(\breve{\xi})$, where $\breve{\xi}=\mathbf{g}(\boldsymbol{\xi})=$ $\left[\delta \boldsymbol{\rho}^{T}\right]^{T}$, by using the change of variable formula (see [12] p. 45)

$$
\operatorname{CRB}(\breve{\xi})=\mathbf{J} \operatorname{CRB}(\boldsymbol{\xi}) \mathbf{J}^{T},
$$

where the separation is given by $\delta=\left|\omega_{1}-\omega_{2}\right|$ and where the Jacobian matrix is given by $[\mathbf{J}]_{i, j}=\frac{\partial[\mathbf{g}(\boldsymbol{\xi})]_{i}}{\partial[\boldsymbol{\xi}]_{j}}$. Consequently, $\mathbf{J}=$ $\left[\begin{array}{cc}\mathbf{h}^{T} & \mathbf{0} \\ \mathbf{0} & \mathbf{I}\end{array}\right]$ where $\mathbf{h}=\operatorname{sgn}\left(\omega_{1}-\omega_{2}\right)[1-1]^{T}$ and $\operatorname{sgn}\left(\omega_{1}-\omega_{2}\right)=$ $\frac{\omega_{1}-\omega_{2}}{\left|\omega_{1}-\omega_{2}\right|}$. Using the Jacobian matrix above and (2), one obtains

$\mathbf{C R B}(\breve{\boldsymbol{\xi}})=\left[\begin{array}{cc}\mathbf{h}^{T} & \mathbf{0} \\ \mathbf{0} & \mathbf{I}\end{array}\right]\left[\begin{array}{ccc}\mathrm{CRB}\left(\omega_{1}\right) & \mathrm{CRB}\left(\omega_{1}, \omega_{2}\right) & \times \\ \mathrm{CRB}\left(\omega_{2}, \omega_{1}\right) & \mathrm{CRB}\left(\omega_{2}\right) & \times \\ \times & \times & \times\end{array}\right]\left[\begin{array}{cc}\mathbf{h} & \mathbf{0} \\ \mathbf{0} & \mathbf{I}\end{array}\right]$ where $\operatorname{CRB}\left(\omega_{i}\right)$ and $\operatorname{CRB}\left(\omega_{1}, \omega_{2}\right) \triangleq[\operatorname{CRB}(\breve{\boldsymbol{\xi}})]_{1,2}$ denote the CRB on $\omega_{i}$ and the cross terms between $\omega_{1}$ and $\omega_{2}$, respectively. Consequently,

$$
\begin{aligned}
& \operatorname{CRB}(\delta) \triangleq \mathbf{C R B}\left([\breve{\xi}]_{1}\right)=\operatorname{sgn}^{2}\left(\omega_{1}-\omega_{2}\right) \operatorname{CRB}\left(\omega_{1}\right)+ \\
& \left(-\operatorname{sgn}\left(\omega_{1}-\omega_{2}\right)\right)^{2} \operatorname{CRB}\left(\omega_{2}\right)-2 \operatorname{sgn}^{2}\left(\omega_{1}-\omega_{2}\right) \operatorname{CRB}\left(\omega_{1}, \omega_{2}\right) \\
& =\operatorname{CRB}\left(\omega_{1}\right)+\operatorname{CRB}\left(\omega_{2}\right)-2 \operatorname{CRB}\left(\omega_{1}, \omega_{2}\right) .
\end{aligned}
$$

From (3) we notice that the SRL using Smith's criterion [3] takes into account the coupling between the parameters of interest. Consequently, using Smith's criterion, the SRL can be re-written as $\delta$ which resolves the following equation

$$
\delta^{2}=\operatorname{CRB}\left(\omega_{1}\right)+\operatorname{CRB}\left(\omega_{2}\right)-2 \operatorname{CRB}\left(\omega_{1}, \omega_{2}\right) .
$$

Finally, note that, as in [7], for the case where the parameters of interest are decoupled, one obtains the SRL by resolving the following equation $\delta^{2}=\operatorname{CRB}\left(\omega_{1}\right)+\operatorname{CRB}\left(\omega_{2}\right)$.

One should note that, unlike Smith's criterion, Lee's criterion ${ }^{1}$ [2] does not take into account the coupling between the parameters that becomes important when the signal parameters are close. In the following section, we will extend the previous SRL to multiple parameters of interest per signal in the case of two emitting signals.

\section{STATISTICAL RESOLUTION LIMIT FOR MULTIPLE PARAMETERS OF INTEREST PER SIGNAL}

Before introducing a scheme to derive the SRL for multiple parameters of interest per signal, we begin by showing that generalizing Smith's approach to derive the SRL for multiple parameters

\footnotetext{
${ }^{1}$ Recall that the SRL based on Lee's criterion [2] is defined as $\delta$ such that $\delta=2 \max \left\{\sqrt{\mathrm{CRB}\left(\omega_{1}\right)}, \sqrt{\mathrm{CRB}\left(\omega_{2}\right)}\right\}$.
}

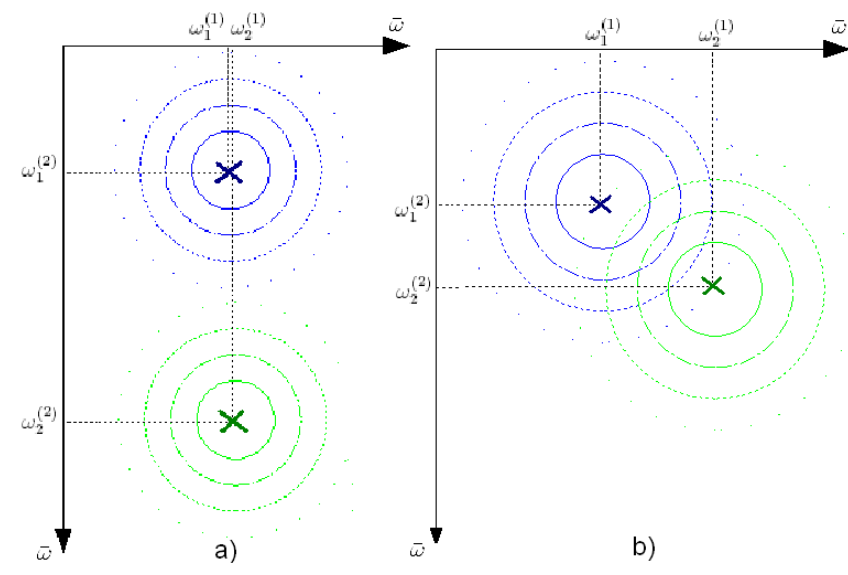

Fig. 1. Localization of two point signals thanks to two parameters of interest where $\bar{\omega}$ denotes the unit of measurement.

is not straightforward. For that purpose, let us consider the simple case of $M=2$ parameters, denoted $\omega_{i}^{(1)}$ and $\omega_{i}^{(2)}$, of interest for the $i^{t h}$ signal. Let $\tilde{\delta}=\left[\begin{array}{ll}\delta_{1} & \delta_{2}\end{array}\right]^{T}$ where $\delta_{1}=\left|\omega_{1}^{(1)}-\omega_{2}^{(1)}\right|$ and $\delta_{2}=\left|\omega_{1}^{(2)}-\omega_{2}^{(2)}\right|$ denote the separation w.r.t. $\omega^{(1)}$ and $\omega^{(2)}$, respectively. Consequently

$$
\tilde{\boldsymbol{\delta}}=\boldsymbol{H} \boldsymbol{\omega} \text { with } \boldsymbol{H}=\left[\begin{array}{cccc}
a_{1} & 0 & -a_{1} & 0 \\
0 & a_{2} & 0 & -a_{2}
\end{array}\right]
$$

where $a_{p}=\operatorname{sgn}\left(\omega_{1}^{(p)}-\omega_{2}^{(p)}\right)$ and $\boldsymbol{\omega}=\left[\omega_{1}^{(1)} \omega_{1}^{(2)} \omega_{2}^{(1)} \omega_{2}^{(2)}\right]^{T}$. From $\operatorname{CRB}(\xi)$, one can deduce $\operatorname{CRB}(\breve{\xi})$ by using the change of variable formula (2), where $\boldsymbol{\xi}=\left[\begin{array}{ll}\boldsymbol{\omega}^{T} & \boldsymbol{\rho}^{T}\end{array}\right]^{T}$ and $\breve{\xi}=\left[\begin{array}{ll}\tilde{\boldsymbol{\delta}}^{T} & \boldsymbol{\rho}^{T}\end{array}\right]^{T}$. The Jacobian matrix is then given by $\mathbf{J}=\left[\begin{array}{cc}\boldsymbol{H} & \mathbf{0} \\ \mathbf{0} & \mathbf{I}\end{array}\right]$. Consequently,

$$
\operatorname{CRB}(\breve{\xi})=\left[\begin{array}{cc}
\boldsymbol{H} & \mathbf{0} \\
\mathbf{0} & \mathbf{I}
\end{array}\right]\left[\begin{array}{cc}
\mathbf{C R B}(\boldsymbol{\omega}) & \times \\
\times & \times
\end{array}\right]\left[\begin{array}{cc}
\boldsymbol{H}^{T} & \mathbf{0} \\
\mathbf{0} & \mathbf{I}
\end{array}\right]
$$

Using the same method as in (4), one obtains

$$
\begin{aligned}
\operatorname{CRB}\left(\delta_{1}\right) \triangleq \mathbf{C R B}\left([\breve{\boldsymbol{\xi}}]_{1}\right)= & \operatorname{CRB}\left(\omega_{1}^{(1)}\right)+\operatorname{CRB}\left(\omega_{2}^{(1)}\right) \\
& -2 \operatorname{CRB}\left(\omega_{1}^{(1)}, \omega_{2}^{(1)}\right),
\end{aligned}
$$

and

$$
\begin{aligned}
\operatorname{CRB}\left(\delta_{2}\right) \triangleq \mathbf{C R B}\left([\breve{\boldsymbol{\xi}}]_{2}\right)= & \operatorname{CRB}\left(\omega_{1}^{(2)}\right)+\operatorname{CRB}\left(\omega_{2}^{(2)}\right) \\
& -2 \operatorname{CRB}\left(\omega_{1}^{(2)}, \omega_{2}^{(2)}\right) .
\end{aligned}
$$

From (5) and (6), we notice that the CRB on the separation w.r.t. $\omega^{(1)}$ is viewed independently from the separation w.r.t. $\omega^{(2)}$ and vice-versa. Consequently, deducing the SRL in the case of multiple parameters of interest per signal using (5) and (6) can be meaningless. As an example, Fig. 1a shows that, thanks to the second parameter of interest, even if $\omega_{1}^{(1)}$ is very close to $\omega_{2}^{(1)}$, the signals can still be well resolvable. However, Fig. $1 \mathrm{~b}$ shows that even if $\omega_{1}^{(1)}$ is not too close to $\omega_{2}^{(1)}$ as in Fig. 1a, the signals might not be resolvable. 


\subsection{Proposed solution}

Let us assume that we have $P$ parameters of interest per signal denoted by $C=\left\{\omega^{(1)}, \omega^{(2)}, \ldots, \omega^{(P)}\right\}$. The question herein addressed is how can we define the SRL such that all the P parameters of interest are taken into account? A natural idea is to consider the distance between the set of the $P$ parameters of interest of the first signal, $C_{1}=\left\{\omega_{1}^{(1)}, \omega_{1}^{(2)}, \ldots, \omega_{1}^{(P)}\right\}$ and the set of the $P$ parameters of interest of the second signal, $C_{2}=\left\{\omega_{2}^{(1)}, \omega_{2}^{(2)}, \ldots, \omega_{2}^{(P)}\right\}$. Let

$$
\delta \triangleq k \text {-norm distance }\left(C_{1}, C_{2}\right)=\left(\sum_{p=1}^{P} \delta_{p}^{k}\right)^{1 / k},
$$

define the SRL w.r.t. the sets $C_{1}$ and $C_{2}$ (such that $C_{1} \neq C_{2}$ ) where $\delta_{p}=\left|\omega_{1}^{(p)}-\omega_{2}^{(p)}\right|$. The $k$-norm distance $\left(C_{1}, C_{2}\right)$ is the so-called Minkowski distance of order $k$. Having $\mathbf{C R B}(\boldsymbol{\xi})$ where $\boldsymbol{\xi}=\left[\boldsymbol{\omega}^{T} \boldsymbol{\rho}^{T}\right]^{T}$ in which

$$
\boldsymbol{\omega}=\left[\omega_{1}^{(1)} \omega_{2}^{(1)} \omega_{1}^{(2)} \omega_{2}^{(2)} \ldots \omega_{1}^{(P)} \omega_{2}^{(P)}\right]^{T},
$$

one can deduce $\mathbf{C R B}(\tilde{\boldsymbol{\xi}})$ where $\tilde{\boldsymbol{\xi}}=\left[\delta \boldsymbol{\rho}^{T}\right]^{T}$. Consequently, the Jacobian matrix is given by $\mathbf{J}=\left[\begin{array}{cc}\mathbf{h}^{T} & \mathbf{0} \\ \mathbf{0} & \mathbf{I}\end{array}\right]$ where

$$
\mathbf{h}=\left[\begin{array}{lllllll}
g_{1} & -g_{1} & g_{2} & -g_{2} & \ldots & g_{P} & -g_{P}
\end{array}\right]^{T},
$$

in which $g_{p}=\frac{\partial \delta}{\partial \omega_{1}^{(p)}}=-\frac{\partial \delta}{\partial \omega_{2}^{(p)}}$. Since $|x|^{k}=\sqrt{x^{2 k}}$ for $x \neq 0$, one has

$$
\begin{aligned}
& g_{p}=\frac{\partial\left(\sum_{q=1}^{P} \sqrt{\left(\omega_{1}^{(q)}-\omega_{2}^{(q)}\right)^{2 k}}\right)^{1 / k}}{\partial \omega_{1}^{(p)}} \\
& =\left(\sum_{q=1}^{P} \sqrt{\left(\omega_{1}^{(q)}-\omega_{2}^{(q)}\right)^{2 k}}\right)^{\frac{1}{k}-1} \sqrt{\left(\omega_{1}^{(p)}-\omega_{2}^{(p)}\right)^{2(k-1)}} \\
& =\delta^{1-k} \delta_{p}^{k-1} .
\end{aligned}
$$

Again, by using the change of variable formula (2), one obtains

$$
\operatorname{CRB}(\tilde{\xi})=\left[\begin{array}{cc}
\mathbf{h}^{T} \mathbf{C R B}(\boldsymbol{\omega}) \mathbf{h} & \times \\
\times & \mathbf{I}
\end{array}\right] .
$$

Consequently, after some calculus, one obtains

$$
\begin{aligned}
& \operatorname{CRB}(\delta) \triangleq \mathbf{C R B}\left([\breve{\xi}]_{1}\right)=\sum_{p=1}^{P} \sum_{q=1}^{P} g_{p} g_{q}\left([\mathbf{C R B}(\boldsymbol{\xi})]_{2 p, 2 q}+\right. \\
& \left.[\mathbf{C R B}(\boldsymbol{\xi})]_{2 p-1,2 q-1}-[\mathbf{C R B}(\boldsymbol{\xi})]_{2 p, 2 q-1}-[\mathbf{C R B}(\boldsymbol{\xi})]_{2 p-1,2 q}\right) \\
& =\delta^{2(1-k)}\left(A_{\text {direct }}+A_{\text {cross }}\right)
\end{aligned}
$$

where $A_{\text {direct }}=\sum_{p=1}^{P} \delta_{p}^{2(k-1)}\left(\operatorname{CRB}\left(\omega_{1}^{(p)}\right)+\operatorname{CRB}\left(\omega_{2}^{(p)}\right)-\right.$ $\left.2 \operatorname{CRB}\left(\omega_{1}^{(p)}, \omega_{2}^{(p)}\right)\right)$ represents the contribution of the parameters of interest for the same parameter $p$ and where $A_{\text {cross }}=$ $\sum_{p=1}^{P} \sum_{\substack{q=1 \\ q \neq p}}^{P} \delta_{p}^{k-1} \delta_{q}^{k-1}\left(\operatorname{CRB}\left(\omega_{1}^{(p)}, \omega_{1}^{(q)}\right)+\operatorname{CRB}\left(\omega_{2}^{(p)}, \omega_{2}^{(q)}\right)-\right.$ $\left.2 \operatorname{CRB}\left(\omega_{1}^{(p)}, \omega_{2}^{(q)}\right)\right)$ represents the contribution of the cross terms between parameters of interest.

Despite of the fact that the 2-norm is the most commonly used norm, it is often more interesting to use the 1 -norm to solve ${ }^{2}$ $\delta^{2}=C R B(\delta)$. Indeed, by doing this, the separation remains linear w.r.t. the parameters. This implies that its first order derivative is parameter independent. In fact, and as expected, if $P=1$ and considering the 1-norm distance, one notices that $g_{1}=1$ and consequently, using (9), one obtains, $\operatorname{CRB}(\delta)=$ $[\mathbf{C R B}(\boldsymbol{\xi})]_{1,1}+[\mathbf{C R B}(\boldsymbol{\xi})]_{2,2}-2[\mathbf{C R B}(\boldsymbol{\xi})]_{1,2}$ which is the same expression as (4).

Remark 1: Let us now consider the case where $P=2$, and let us assume, for sake of simplicity, that the parameters $\omega_{1}^{(p)}$ and $\omega_{2}^{(q)}$ $\forall p, q$ are decoupled. Applying (9) one obtains,

$$
\begin{aligned}
& \operatorname{CRB}(\delta)=\delta^{2(1-k)}\left[\delta_{1}^{2(k-1)}\left(\operatorname{CRB}\left(\omega_{1}^{(1)}\right)+\operatorname{CRB}\left(\omega_{2}^{(1)}\right)\right)\right. \\
& +\delta_{2}^{2(k-1)}\left(\operatorname{CRB}\left(\omega_{1}^{(2)}\right)+\operatorname{CRB}\left(\omega_{2}^{(2)}\right)\right) \\
& \left.+2 \delta_{1}^{k-1} \delta_{2}^{k-1}\left(\operatorname{CRB}\left(\omega_{1}^{(1)}, \omega_{1}^{(2)}\right)+\operatorname{CRB}\left(\omega_{2}^{(1)}, \omega_{2}^{(2)}\right)\right)\right] .
\end{aligned}
$$

We notice that, unlike (5) and (6), equation (10) takes into account the effect of parameters of different nature thanks to the cross terms $\operatorname{CRB}\left(\omega_{i}^{(1)}, \omega_{j}^{(2)}\right)$.

Remark 2: In the case where we are interested by deriving the SRL w.r.t. multiple physical parameters of interest having different units of measurement, we cannot use directly formula (9). To illustrate how to derive the SRL in this case, we consider for instance the problem of the localisation of two near-field sources parameterized by two physical parameters, namely the bearing $\theta$ in radian and the range $r$ in meter. Toward the derivation of the SRL, we have to

1. Derive the CRB w.r.t. to the physical parameters corresponding to $\left[\begin{array}{lllll}\theta_{1} & \theta_{2} & r_{1} & r_{2} & \boldsymbol{\rho}^{T}\end{array}\right]^{T}$.

2. Deduce the CRB w.r.t. to the non-physical parameters corresponding to $\left[\omega_{1}^{(1)} \omega_{2}^{(1)} \omega_{1}^{(2)} \omega_{2}^{(2)} \rho^{T}\right]^{T}$ thanks to a proper change of variable. This change of variable is deduced from the definition of the electric angles $\omega_{i}^{(1)}=-2 \pi d / \lambda \sin \left(\theta_{i}\right)$ and $\omega_{i}^{(2)}=\pi^{2}\left(d^{2} / \lambda\right) \cos ^{2}\left(\theta_{i}\right) / r_{i}$ where $d$ is the distance inter-sensor and $\lambda$ is the signal wavelength [13].

3. Choose $k$ and deduce the $\operatorname{CRB}(\delta)$ where $\delta$ is defined in (7) using formula (9).

4. Finally, solve the implicit equation $\delta^{2}=\mathrm{CRB}(\delta)$ which provides the SRL.

In the following, this result is extended to the case of $M \geq 2$ signals where each signal is parameterized by $P$ parameters of interest per signal.

\section{STATISTICAL RESOLUTION LIMIT FOR MULTIPLE SIGNALS}

We begin by deriving the SRL for each couple of signals. Using the Newton's binomial theorem for $M$ signals, the number of signal's couples is equal to $\frac{M(M-1)}{2}$. Then, the SRL will be the worst SRL, i.e., the maximum of all the minimal distances between each couple of two closely spaced signals that allows a correct resolvability.

${ }^{2}$ However, if $\exists p \in[1 \ldots P]$ such that $\omega_{1}^{(p)}=\omega_{2}^{(p)}$, then $\frac{\partial \delta}{\partial \omega_{1}^{(p)}}$ exists only if the $k$-norm distance is such that $k$ is an even number. 
From $\mathbf{C R B}(\boldsymbol{\xi})$, one can deduce $\mathbf{C R B}(\breve{\boldsymbol{\xi}})$ using the change of variable formula (2) where $\boldsymbol{\xi}=\left[\begin{array}{ll}\boldsymbol{\omega}^{T} & \boldsymbol{\rho}^{T}\end{array}\right]^{T}$ with

$$
\boldsymbol{\omega}=\left[\begin{array}{lll}
\left(\boldsymbol{\omega}^{(1)}\right)^{T} & \cdots & \left(\boldsymbol{\omega}^{(P)}\right)^{T}
\end{array}\right]^{T}
$$

in which $\boldsymbol{\omega}^{(p)}=\left[\omega_{1}^{(p)} \omega_{2}^{(p)} \ldots \omega_{M}^{(p)}\right]^{T}$ and $\breve{\boldsymbol{\xi}}=\mathbf{g}(\boldsymbol{\xi})=\left[\boldsymbol{\delta}^{T} \boldsymbol{\rho}^{T}\right]^{T}$

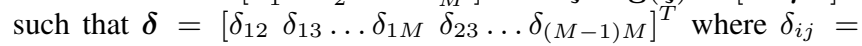
$\left(\sum_{p=1}^{P}\left(\delta_{p}^{(i j)}\right)^{k}\right)^{1 / k}$ and $\delta_{p}^{(i j)}=\left|\omega_{i}^{(p)}-\omega_{j}^{(p)}\right|$. The separation $\delta_{i j}$ is the $k$-norm distance between the $i^{\text {th }}$ and the $j^{\text {th }}$ signal. Thus, the Jacobian matrix is given by $\mathbf{J}=\left[\begin{array}{cc}\boldsymbol{H} & \mathbf{0} \\ \mathbf{0} & \mathbf{I}\end{array}\right]$ in which $\boldsymbol{H}$ is a $\left(\frac{M(M-1)}{2}\right) \times(M P)$ matrix given by $\boldsymbol{H}=$ $\left[\begin{array}{llll}\boldsymbol{\alpha}_{12} & \boldsymbol{\alpha}_{13} & \ldots & \boldsymbol{\alpha}_{(M-1) M}\end{array}\right]^{T}$ where $\boldsymbol{\alpha}_{i j}=\left[\begin{array}{lll}\boldsymbol{\eta}_{i j 1}^{T} & \ldots & \boldsymbol{\eta}_{i j P}^{T}\end{array}\right]^{T}$ in which

$$
\left[\boldsymbol{\eta}_{i j p}\right]_{q}= \begin{cases}\frac{\partial \delta_{i j}}{\partial \omega_{i}^{(p)}} & \text { for } q=i \\ -\frac{\partial \delta_{i j}}{\partial \omega_{i}^{(p)}} & \text { for } q=j \\ 0 & \text { otherwise }\end{cases}
$$

where $\frac{\partial \delta_{i j}}{\partial \omega_{i}^{(p)}}=\delta_{i j}^{1-k}\left(\delta_{p}^{(i j)}\right)^{k-1}$.

We have $\mathbf{C R B}(\breve{\xi})=\left[\begin{array}{cc}\boldsymbol{H} \mathbf{C R B}(\boldsymbol{\omega}) \boldsymbol{H}^{T} & \times \\ \times & \mathbf{I}\end{array}\right]$. Finally, taking into account only the main diagonal terms, one obtains

$$
\begin{aligned}
& \operatorname{CRB}\left(\delta_{i j}\right)=\sum_{p=1}^{M P} \sum_{q=1}^{M P}\left[\boldsymbol{\alpha}_{i j}\right]_{p}\left[\boldsymbol{\alpha}_{i j}\right]_{q}[\mathbf{C R B}(\boldsymbol{\xi})]_{p, q} \\
& =\sum_{p=1}^{P} \sum_{q=1}^{P} \frac{\partial \delta_{i j}}{\partial \omega_{i}^{(p)}} \frac{\partial \delta_{i j}}{\partial \omega_{i}^{(q)}}\left([\mathbf{C R B}(\boldsymbol{\xi})]_{i+M(p-1), i+M(q-1)}+\right. \\
& {[\mathbf{C R B}(\boldsymbol{\xi})]_{j+M(p-1), j+M(q-1)}-[\mathbf{C R B}(\boldsymbol{\xi})]_{i+M(p-1), j+M(q-1)}} \\
& \left.-[\mathbf{C R B}(\boldsymbol{\xi})]_{j+M(p-1), i+M(q-1)}\right) \\
& =\delta_{i j}^{2(1-k)}\left(A_{\text {direct }}^{(i j)}+A_{\text {cross }}^{(i j)}\right),
\end{aligned}
$$

where $A_{\text {direct }}^{(i j)}=\sum_{p=1}^{P}\left(\delta_{p}^{(i j)}\right)^{2(k-1)}\left(\operatorname{CRB}\left(\omega_{i}^{(p)}\right)+\operatorname{CRB}\left(\omega_{j}^{(p)}\right)\right.$ $\left.-2 \mathrm{CRB}\left(\omega_{i}^{(p)}, \omega_{j}^{(p)}\right)\right)$ represents the contribution of the parameters of interest for the same $p$ and for the $i^{t h}$ and $j^{\text {th }}$ signals and

$$
\begin{aligned}
& A_{\text {cross }}^{(i j)}=\sum_{p=1}^{P} \sum_{\substack{q=1 \\
q \neq p}}^{P}\left(\delta_{p}^{(i j)}\right)^{k-1}\left(\delta_{q}^{(i j)}\right)^{k-1} \times \\
& \left(\operatorname{CRB}\left(\omega_{i}^{(p)}, \omega_{i}^{(q)}\right)+\operatorname{CRB}\left(\omega_{j}^{(p)}, \omega_{j}^{(q)}\right)-2 \operatorname{CRB}\left(\omega_{i}^{(p)}, \omega_{j}^{(q)}\right)\right),
\end{aligned}
$$

represents the contribution of the cross terms between parameters of interest for the $i^{t h}$ and $j^{t h}$ signals. Using (11) one can deduce the SRL as the maximum SRL for each couple of signals, i.e.,

$$
\delta=\max \left\{\delta_{i j} \text { for } i<j \text { and } i, j \leq M\right\} .
$$

One should note that even if we derive the SRL for each couple of signals, we are also taking into account the influence of the other signals thanks to the use of the CRB regarding to the full vector of parameters $\boldsymbol{\xi}$. As an example, for $M=2$, applying (11) one obtains (9). And for $M=2, P=1$ and $k=1$ one obtains the equivalent Smith's equation written in (4).

\section{CONCLUSION}

In this paper, we extended the Statistical Resolution Limit to multiple parameters of interest per signal and multiple signals. Toward this end, we give a fresh look at Smith's criterion and defined an $e x-$ tended SRL thanks to the Minkowski distances of order $k$. By using proper changes of variable formula, we obtain general results on the SRL for multiple parameters of interest per signal and multiple signals. The results presented herein can be applied to any estimation problem and are not limited to the source localization problems.

\section{REFERENCES}

[1] H. L. VanTrees, Detection, Estimation and Modulation Theory. New York: Wiley, 1968, vol. 1.

[2] H. B. Lee, "The Cramér-Rao bound on frequency estimates of signals closely spaced in frequency," IEEE Trans. Signal Processing, vol. 40, no. 6, pp. 1507-1517, 1992.

[3] S. T. Smith, "Statistical resolution limits and the complexified Cramér Rao bound," IEEE Trans. Signal Processing, vol. 53, pp. 1597-1609, May 2005.

[4] A. Amar and A. Weiss, "Fundamental limitations on the resolution of deterministic signals," IEEE Trans. Signal Processing, vol. 56, no. 11, pp. 5309-5318, Nov. 2008.

[5] E. Dilaveroglu, "Nonmatrix Cramér-Rao bound expressions for high-resolution frequency estimators," IEEE Trans. Signal Processing, vol. 46, no. 2, pp. 463-474, Feb. 1998.

[6] D. Swingler, "Frequency estimation for closely spaced sinsoids: Simple approximations to the Cramér-Rao lower bound," IEEE Trans. Signal Processing, vol. 41, no. 1, pp. 489495, Jan. 1993.

[7] J.-P. Delmas and H. Abeida, "Statistical resolution limits of DOA for discrete sources," in Proc. IEEE Int. Conf. Acoust., Speech, Signal Processing, vol. 4, Toulouse, France, 2006, pp. 889-892.

[8] E. Grosicki, K. Abed-Meraim, and Y. Hua, "A weighted linear prediction method for near-field source localization," IEEE Trans. Signal Processing, vol. 53, pp. 3651-3660, 2005.

[9] J. Li, P. Stoica, and D. Zheng, "Efficient direction and polarization estimation with a cold array," IEEE Trans. Antennas Propagat., vol. 44, no. 4, pp. 539-547, Apr. 1996.

[10] L. Godara, "Applications of antenna arrays to mobile communications: II. beam-forming and direction of arrival considerations," IEEE Trans. Antennas Propagat., vol. 85, no. 8, pp. 1195-1245, Aug. 1997.

[11] H. Cramér, Mathematical Methods of Statistics. New York: Princeton University, Press, 1946.

[12] S. M. Kay, Fundamentals of Statistical Signal Processing. NJ: Prentice Hall, 1993, vol. 1.

[13] M. N. El Korso, R. Boyer, A. Renaux, and S. Marcos, "Nonmatrix closed-form expressions of the Cramér-Rao bounds for near-field localization parameters," in Proc. IEEE Int. Conf. Acoust., Speech, Signal Processing, Taipei, Taiwan, 2009, pp. 3277-3280. 